\title{
The Effects of Gene Variations of GABRA2, GABRBI, GABRG2, GADI and SLCIA3 on Patients with Propofol During Anesthesia Induction
}

\author{
Lingyi Zhang, ', Zhuoling Zheng, 2,* \\ Wudi Ma,' Shuyu Zhang, \\ Faling Xue, ' Haini Wang, \\ Yongqi He, ${ }^{6}$ Fang Ye, \\ Shouning Zhou, ${ }^{6}$ Yongzi Wen, ${ }^{3,4}$ \\ Xiaoyan Li, ${ }^{2}$ Wenqi Huang,' \\ Min Huang, 3,4 Jiali Li, ${ }^{3,4, *}$ \\ Zhongxing Wang (D) ${ }^{1, *}$
}

'Department of Anesthesiology, The First Affiliated Hospital of Sun Yat-sen University, Guangzhou, Guangdong, People's Republic of China; ${ }^{2}$ Department of Pharmacy, The Sixth Affiliated Hospital of Sun Yat-Sen University, Guangzhou, Guangdong, People’s Republic of China; ${ }^{3}$ Institute of Clinical Pharmacology, School of Pharmaceutical Sciences, Sun Yat-sen University, Guangzhou, Guangdong, People's Republic of China;

${ }^{4}$ Guangdong Provincial Key Laboratory of

New Drug Design and Evaluation, School of Pharmaceutical Sciences, Sun Yat-sen

University, Guangzhou, Guangdong, People’s Republic of China; ${ }^{5}$ Department of Pharmacy, Shenzhen Second People's Hospital, Shenzhen, Guangdong, People's Republic of China; ${ }^{6}$ Department of Pharmacy, The First Affiliated Hospital of Guangzhou Medical University, Guangzhou, Guangdong, People's Republic of China

*These authors contributed equally to this work

Correspondence: Zhongxing Wang Department of Anesthesiology, The First Affiliated Hospital of Sun Yat-sen University, 58 Zhongshan Road 2, Guangzhou, 510080 ,

People's Republic of China

Tel +86-20-87755766

Email wzhxing@mail.sysu.edu.cn

Jiali $\mathrm{Li}$

Institute of Clinical Pharmacology, School of Pharmaceutical Sciences, Sun Yat-sen University, I 32 Waihuan Dong Road, University City, Guangzhou, 510006,

People's Republic of China

Tel +86-20-39943034

Email lijiali5@mail.sysu.edu.cn
Purpose: Propofol is one of the most commonly used intravenous sedatives in general anesthesia, while the individual variations of propofol are apparent. The objective of this study was to investigate the influence of genetic variations in GABAergic neurons and glutamatergic neurons on time to loss of consciousness (LOC) and the incidence of hypotension during anesthesia induction.

Patients and Methods: A total of 140 Chinese patients undergoing thyroid surgery or breast surgery were recruited. Genotyping of candidate genes was carried out using the Agena Bioscience MassARRAY system. Anesthesia induction was initiated with a propofol target plasma concentration $(\mathrm{Cp})$ of $4.0 \mu \mathrm{g} \mathrm{mL}^{-1}$. The LOC latency, systolic blood pressure, diastolic blood pressure, mean arterial pressure were documented.

Results: We found that GABRA2 rs35496835, GABRB1 rs1372496, GABRG2 rs11135176, GABRG2 rs209358, GAD1 rs3791878, SLC1A3 rs1049522 and gender were significant determinants of the patient's LOC latency following propofol administration. GABRA2 rs11503014 was highly correlated with blood pressure reduction during anesthesia induction. Multiple linear regression analysis revealed that GABRB1 rs1372496, GABRG2 rs11135176, and SLC1A3 rs 1049522 accounted for $35.3 \%$ variations in LOC latency following propofol administration.

Conclusion: Our findings indicate that genetic variants of GABRA2, GABRB1, GABRG2, GAD1 and SLC1A3 may have influence on propofol susceptibility, which would be an important guidance towards building clinical models that can precisely predict the efficacy of propofol with various populations before surgery.

Keywords: propofol, anesthesia, GABRB1, LOC latency

\section{Introduction}

Propofol is one of the most commonly used intravenous agents for both the induction and maintenance of total intravenous general anesthesia. ${ }^{1,2}$ The targetcontrolled infusion (TCI) of propofol has been widely studied and applied in clinical settings. ${ }^{3,4}$ However, the patients' responses to propofol vary significantly, even under the same target concentration. It had been shown that the time to loss of verbal contact and the time to bispectral index $<70$ varied by 6.6 - and 4.3 -fold, respectively. ${ }^{5}$ Improper level of sedation may lead to prolonged induction time, hypotension, bradycardia and respiratory depression. ${ }^{6,7}$ Therefore, it is essential to study the individual differences of response related to propofol among patients and identify some biomarkers for the prediction of propofol susceptibility. 
Propofol promotes gamma-aminobutyric acid type A receptor (GABAAR)-mediated chloride conductance, which enhances inhibitory post-synaptic potentials. ${ }^{8}$ In addition, propofol inhibits the action of glutamatemediated excitatory nerves. ${ }^{9,10}$ Individual differences of propofol are attributed to many factors, including genetic polymorphisms. Nowadays, only a few pharmacogenetic studies have focused on the significant inter-individual variances in propofol susceptibility or the effects on GABAergic neurons and glutamatergic neurons. Zhong ${ }^{11}$ found that Sodium Voltage-Gated Channel Alpha Subunit 9 (SCN9A) rs6746030 was associated with the increased response to propofol, and that Dopamine Receptor D2 (DRD2) rs2283265 was associated with cardiovascular susceptibility to propofol anesthesia. IohomG ${ }^{5}$ analysed the correlation between Gamma-aminobutyric Acid Type A Receptor Subunit Epsilon (GABRE) [mRNA358] G/T, $20118 \mathrm{C} / \mathrm{T}, 20326 \mathrm{C} / \mathrm{T}$ and $20502 \mathrm{~A} / \mathrm{T}$ and individual differences of propofol, while no positive results were found. Therefore, whether gene polymorphisms in GABAergic neurons and glutamatergic neurons would affect the response to propofol in the clinic remain unknown.

In order to provide more comprehensive evidences regarding individualized use of propofol, this study investigated the effects of genetic variations in GABAergic and glutamatergic neurons on the responses to propofol in 140 Chinese patients. Time to loss of consciousness (LOC) and incidence of hypotension during anesthesia induction were selected as candidate physiologic phenotypes of responses related to propofol.

\section{Materials and Methods Study Design}

The study complied with the Declaration of Helsinki protocol and was approved by the Ethics Committee of the First Affiliated Hospital of Sun Yat-sen University, Guangzhou, China. Patient enrolment, sample collection and follow-up were registered at the Chinese Clinical Trial Registry (ChiCTR1900022251). Written informed consent was obtained from all participants. In patients undergoing total intravenous anesthesia with propofol, we previously observed that the sleep latency was about $(1.60 \pm 0.80$, median \pm interquartile range) min. SNP (single nucleotide polymorphism) that analysed in our study had $>0.10$ minor allele frequency in Chinese population. With a two-tailed alpha of 0.05 and a power of 0.80 , this research was designed to detect a difference between groups of at least
$0.50 \mathrm{~min}$ in sleep latency. Based on these estimates, a total of 108 patients were required for this study. Finally, a total of 140 patients undergoing thyroid surgery or breast surgery under total intravenous anesthesia with propofol were enrolled in this study.

The inclusion criteria were as follows: (1) male or female patients over 18 years old, (2) patients with American Society of Anesthesiologists (ASA) ${ }^{12}$ physical status class I to II, and (3) patients initially scheduled for thyroid surgery or breast surgery. The exclusion criteria were as follows: (1) patients with serious underlying diseases (heart failure, severe liver insufficiency, severe renal insufficiency, endocrine disorders, or any other condition that fitted the ASA III-V category). (2) patients with long-term medication history of sedatives, and (3) patients using cytochrome P450 modulators within one month.

\section{Anesthesia Management}

Patients under total intravenous anesthesia were administered with propofol (Diprifusor, AstraZeneca, England). In accordance with the Marsh's pharmacokinetic models, ${ }^{13}$ a TCI pump of propofol (Injectomat TIVA Agilia, Fresenius, Germany) was used for the induction and maintenance of anesthesia. Anesthetic induction was initiated with a propofol target plasma concentration $(\mathrm{Cp})$ of 4.0 $\mu \mathrm{g} \mathrm{mL}^{-1}$. The Narcotrend system (Narcotrend-Compact, MT MonitorTechnik GmbH \& Co. KG, Hannover Medical School, Germany) provided anesthesia depth classified from A (awake) to F (excessive anesthesia/burst suppression). To minimize the effects induced by dose difference of propofol on the study results, the Cp was increased only when the patient was unable to lose consciousness with the target concentration of $4 \mu \mathrm{g} \mathrm{mL} \mathrm{m}^{-1}$. Opioids and muscle relaxants were used right after the time of LOC to avoid interference in the assessment of LOC. The time of LOC was defined as loss of verbal commands and eyelash reflex. LOC latency, defined as the time from induction initiation to LOC, was documented. TCI was guided by a Narcotrend anesthetic depth monitor after LOC. Systolic blood pressure (SP), diastolic blood pressure (DP), and mean arterial pressure (MAP) were recorded at two time points (before any sedative prescriptions and at the time of LOC). Hypotension was defined as the SP reduction of over $20 \%$ at the time of LOC compared with that at the baseline. 


\section{DNA Isolation and Genotyping}

Peripheral whole-blood sample of each patient was collected in EDTA-coated polypropylene tubes and centrifuged at $4000 \mathrm{rpm}$ for $10 \mathrm{~min}$. DNA isolation was conducted with the improved phenol-chloroform extraction method. ${ }^{14}$ All blood and DNA samples were frozen at $-80^{\circ} \mathrm{C}$ until analysis. Important genes in GABAergic and glutamatergic neurons were explored in this study. Firstly, SNPs of the candidate genes, with minor allele frequencies over 0.10 in Chinese population, were selected by the NCBI (https://www.ncbi.nlm.nih.gov/) and Ensembl (http://asia.ensembl.org/index.html) databases. Then, we searched PubMed and Embase for researches related to these SNPs. SNPs that had been reported in previous studies ${ }^{15-21}$ were preferred. Finally, a total of 15 SNPs, including SNPs in Gamma-Aminobutyric Acid Type A Receptor Subunit Alpha 2 (GABRA2; rs11503014, rs279827, rs279858, rs35496835), GammaAminobutyric Acid Type A Receptor Subunit Beta 1 (GABRB1; rs6290, rs1372496, rs4627835, rs16860087), Gamma-Aminobutyric Acid Type A Receptor Subunit Gamma 2 (GABRG2; rs211037, rs11135176, rs209358), Glutamate Decarboxylase 1 (GAD1; rs3749034, rs3791878) and Solute carrier family 1 member 3 (SLC1A3; rs1049524, rs1049522), were genotyped using the Agena MassArray Analyzer 4 system (Agena, USA).

\section{Statistical Analysis}

Statistical analysis was performed using SPSS version 22.0 (IBM, USA). The data of LOC latency were abnormal distribution and expressed as the median and interquartile range. The Mann-Whitney $U$-test or KruskalWallis test was applied to assess the relationship between genetic polymorphisms and LOC latency. Chi-square test was conducted to examine the correlation between the genotypes and blood pressure changes. Spearman correlation analysis or Chi-square test was performed to analyse the correlation between demographic characteristics and LOC latency. Statistical significance was defined as $P$ values $<0.05$. The SNPstats software (http://bioinfo. iconcologia.net/SNPstats) was used to analyse the SNP model, which was estimated by the following genetic models: the codominant, dominant, recessive, overdominant, and log-additive models. When the Akaike information criterion and Bayesian information criterion were the lowest, the genetic model was selected as the optimal model for the SNP. SNP functions were predicted by the SNPinfo Web Server (https://snpinfo.niehs.nih.gov/ snpinfo/snpfunc.html). With consideration of confounding factors, such as gender, age, weight, height, ASA and baseline biochemical indicators, statistical significance had been reanalysed by multivariate linear regression. The GraphPad Prism 5 (GraphPad, USA) software was used for drawing plots.

\section{Results}

\section{Patients' Characteristics}

With the same initial TCI concentration of propofol, considerable inter-individual differences in LOC latency were recorded in this study. The time from initiation of induction to LOC ranged from $0.50 \mathrm{~min}$ to $11.00 \mathrm{~min}$, with a median of $1.62 \mathrm{~min}$. Supplementary Tables 1 and 2 summarize the demographic characteristics of the recruited patients and the correlation between demographic information and LOC latency induced by propofol. Only gender was associated with LOC latency. Female patients required a shorter time to LOC than male patients $(1.58+1.13 \mathrm{~min}$ vs $2.10+1.16 \mathrm{~min}, P=0.025)$.

\section{Correlation Between Polymorphisms and Propofol Susceptibility}

The allelic frequencies and genotype distributions of the recruited patients are shown in Supplementary Table 3. All SNPs were in the Hardy-Weinberg equilibrium. Linkage disequilibrium analysis showed that linkage disequilibrium existed in GABRA2 rs279827 and rs279858 (D'=1.00, $\mathrm{r}^{2}=0.82$ ).

Among all the detected SNPs, GABRA2 rs35496835, GABRB1 rs1372496, GABRG2 rs11135176, GABRG2 rs209358, GAD1 rs3791878 and SLC1A3 rs1049522 were significant determinants of LOC latency induced by propofol (Figure 1). Patients with the following genotypes had a significantly longer LOC latency than others: GABRA2 rs35496835 GG+G.del genotype (GG+G.del vs del.del, 1.90 +1.03 vs $1.33+0.71 \mathrm{~min}, P=0.010), G A B R B 1$ rs $1372496 \mathrm{CC}$ genotype (TT+TC vs $\mathrm{CC}, 1.39+0.84$ vs $1.83+1.13 \mathrm{~min}$, $P=0.002$ ), $G A B R G 2$ rs $11135176 \mathrm{CC}$ genotype (CC vs CT +TT, $1.75+1.17$ vs $1.50+1.00 \mathrm{~min}, P=0.035), G A B R G 2$ rs209358 TT genotype $(\mathrm{CC}+\mathrm{CT}$ vs TT, $1.50+0.95$ vs 2.00 $+1.09 \mathrm{~min}, P=0.031)$, GAD1 rs3791878 GT+TT genotype (GG vs GT+TT, $1.50+1.09$ vs $1.87+1.01 \mathrm{~min}, P=0.030$ ), SLC1A3 rs1049522 AC genotype (AA+CC vs AC, 1.50 +1.15 vs $1.83+1.22 \mathrm{~min}, P=0.039$ ).

With regard to cardiovascular susceptibility, GABRA2 rs11503014 was significantly associated with SP reduction (GG+GC vs CC, $53 \%$ vs $25 \%, P=0.035$ ), DP reduction (GG 

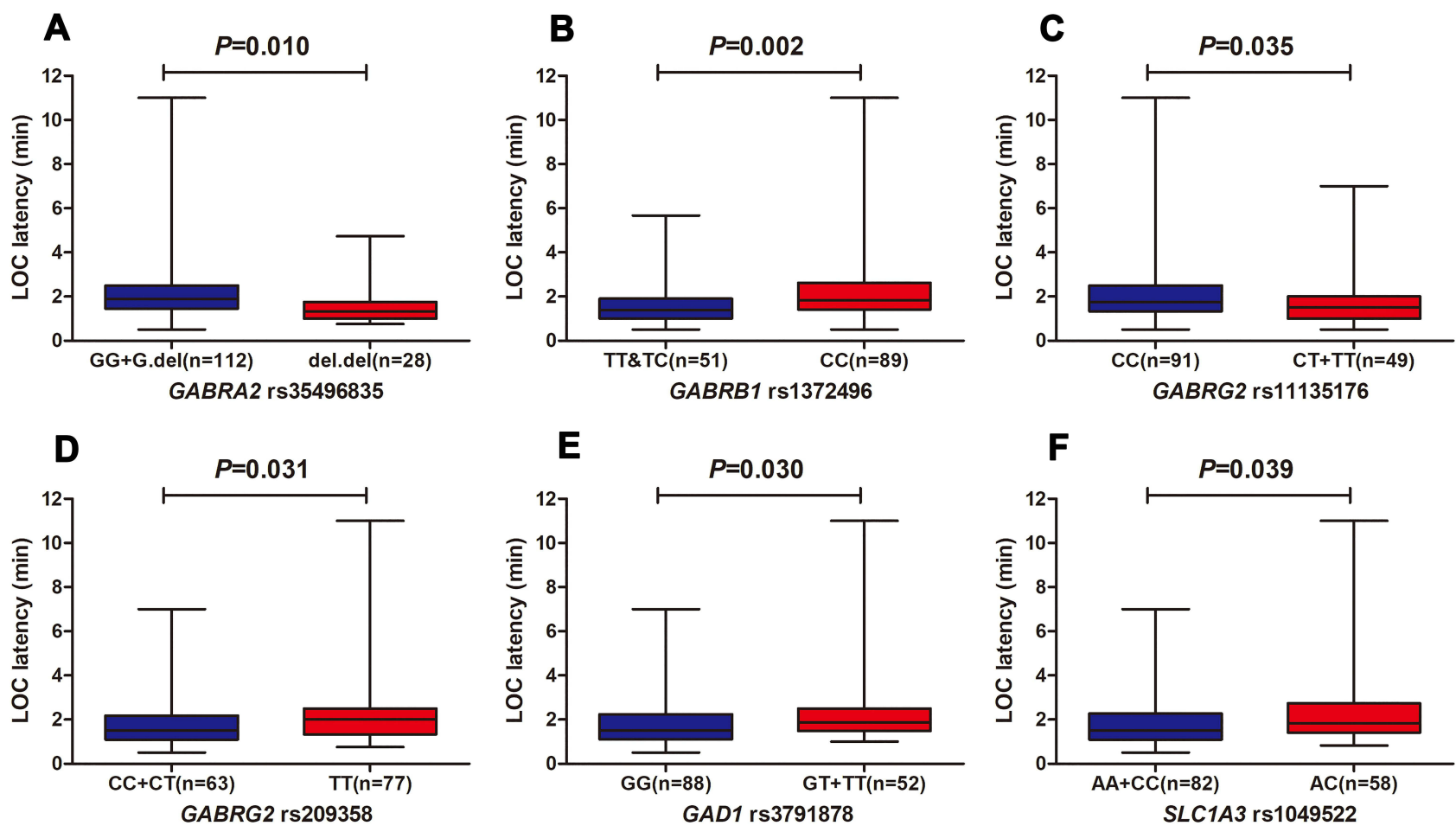

Figure I Association of LOC latency with SNPs. (A) GABRA2 rs35496835, (B) GABRBI rs 1372496, (C) GABRG2 rs I II35 I76, (D) GABRG2 rs209358, (E) GADI rs379|878, (F) SLCIA3 rs 1049522. Error bars represent median and range.

$+\mathrm{GC}$ vs $\mathrm{CC}, 60 \%$ vs $27 \%, P=0.030$ ), and MAP reduction (GG+GC vs $\mathrm{CC}, 53 \%$ vs $24 \%, P=0.016$ ). Compared with the CC genotype of GABRA2 rs11503014, the GG+GC genotype increased the risk of hypotension (Figure 2).

\section{Multivariate Linear Analysis of SNPs with LOC Latency Accounting for Potential Confounding Factors}

To evaluate the effect of genotypes and demographic characteristics on LOC latency, multiple variable analysis was carried out. The final model accounted for $35.3 \%$ of the individual variation in LOC latency induced by propofol, including SNPs of GABRB1 rs1372496, GABRG2 rs11135176, and SLC1A3 rs1049522. Among these genes, GABRG2 rs11135176 contributed the most to the inter-individual variability in LOC latency, with a proportion of $22.2 \%$, whereas GABRB1 rs1372496 and SLC1A3 rs1049522 were responsible for interindividual variabilities of $20.2 \%$ and $12.7 \%$, respectively (Table 1).

\section{Discussion}

Propofol is one of the most commonly used intravenous agents for both the induction and maintenance of total intravenous general anesthesia. However, the significant
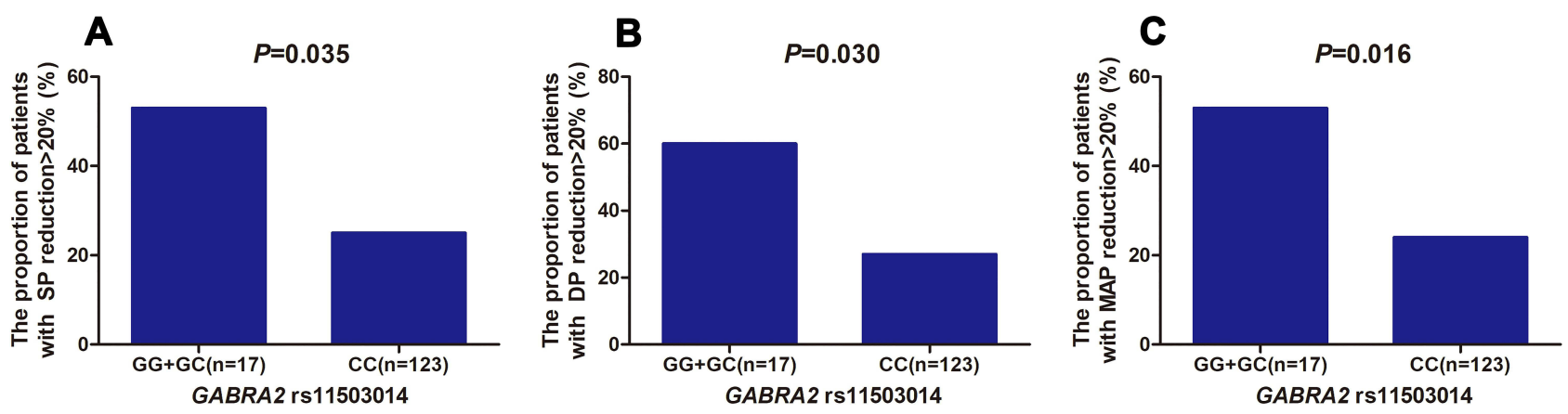

Figure 2 Association of hypotension with GABRA2 rsII5030I4. GABRA2 rsII5030I4 was significantly associated with SP reduction (A), DP reduction (B), and MAP reduction $(\mathbf{C})$ in the recessive model.

Abbreviations: SP, systolic blood pressure; DP, diastolic blood pressure; MAP, mean arterial pressure. 
Table I Multiple Linear Regression Analysis of LOC Latency

\begin{tabular}{|c|c|c|c|c|c|}
\hline Variable & Unstandardized Coefficients & Standardized Coefficients & $P$ values & Partial $r$ & Partial $r^{2}$ \\
\hline (Constant) & 1.191 & - & $<0.001$ & - & - \\
\hline GABRBI rsI372496 & -0.783 & -0.396 & 0.006 & -0.449 & 0.202 \\
\hline GABRG2 rs III35I76 & 0.909 & 0.431 & 0.004 & 0.471 & 0.222 \\
\hline SLCIA3 rs 1049522 & 0.549 & 0.304 & 0.033 & 0.356 & 0.127 \\
\hline
\end{tabular}

Notes: In this equation, genotypes were be assigned as: (I) GABRBI rsI372496, TT+TC=I, CC=0; (2) GABRG2 rs III35I76, CC=I, CT+TT=0; (3) SLCIA3 rs I049522, $A C=I, A A+C C=0$.

inter-individual differences in LOC latency suggested that even under administration at the same TCI concentration, the pharmacodynamic effects of propofol varied significantly among patients. In this study, we found, for the first time, that GABRA2, GABRB1, GABRG2, GAD1 and $S L C 1 A 3$ gene polymorphisms were associated with LOC latency during propofol mediated anesthesia. GABRA2 rs11503014 was significantly associated with hypotension during anesthesia induction. Multiple regression analysis showed that GABRB1 rs1372496, GABRG2 rs11135176, and SLC1A3 rs1049522 accounted for $35.3 \%$ of the individual variation in LOC latency induced by propofol.

Propofol exerts its hypnotic effect through potentiating the inhibitory neurotransmitter GABA. The action of GABA on GABAAR is modulated either by prolonging inhibitory postsynaptic currents mediated by GABAA receptors or by enhancing GABA release via presynaptic mechanisms. ${ }^{22,23}$ GABAAR is a member of the ligand-gated ion channel superfamily, which consists of various subunits $(\alpha 1-6, \beta 1-4$, $\gamma 1-3, \delta, \varepsilon$, and $\rho 1-3) .{ }^{24}$ Anesthetics can bind to hydrophobic pockets within different GABAA receptor subunits ${ }^{25}$ to increase the chloride influx current and neuronal hyperpolarization, and propofol mostly binds on the $\beta$ subunits.

However, no significant finding has been reported about the influence of GABAAR polymorphisms on response related to propofol among patients until now. In our study, GABRB1 rs1372496, the SNP of $\beta 1$ subunit, was found to be correlated with the patients' LOC latency after propofol administration and also included in the multiple linear regression model. GABRB1 rs1372496 is located in the intron region, and the intronic gene polymorphisms are associated with drug susceptibility for their affection on the alternative splicing of human genes. $^{26-28}$ Mutation of GABRB1 rs1372496 may have close relationship with the structure and function of GABAAR $\beta 1$ subunit and the combination of propofol and GABAAR. Meanwhile, different subunit compositions give rise to subtly different subtypes of GABAA receptor, which may also bind to propofol. Our study found that polymorphisms of GABAAR $\alpha$ and $\gamma$ subunit were also associated with LOC latency during propofol mediated anesthesia. GABRA2 and GABRG2 encoded the $\alpha 2$ and $\gamma 2$ subunits of GABAAR, respectively, and we found that $G A B R A 2$ rs35496835, GABRG2 rs11135176 and $G A B R G 2$ rs209358 were correlated with the LOC latency for the first time. GABRG2 rs11135176 was also included in multiple linear regression model for predicting the LOC latency of patients anesthetized with propofol. It was suggested that as a synonymous mutation, GABRG2 rs11135176 might bind to an exonic splicing enhancer or silencer, thereby affecting the cleavage of mRNA and functions of the gene.

Although the inhibiting effect of propofol on central nervous system is probably mediated through the GABA receptors, other pathways may also play a role. In our study, SLC1A3 rs1049522 and GAD1 rs3791878 were found to be associated with LOC latency. Propofol can inhibit the $\mathrm{Na}^{+}$channel-mediated glutamate release. ${ }^{9}$ SLC1A3, a type of glutamate transporter, played a key role in the rapid removal of glutamate released from the synaptic cleft. ${ }^{29}$ SLC1A3 rs1049522 was predicted to be a binding site for microRNAs (miRNAs) in the SNP function prediction website. Furthermore, GAD was the rate-limiting enzyme in GABA formation. $^{30}$ It had been reported that GAD1 rs3791878 and its haplotypes (rs3762556 [C] rs3791878 [G] rs6755102 [C]) were associated with schizophrenia in the Chinese Han population. ${ }^{31}$ Therefore, further studies should pay more attention to the influence of polymorphisms of SLC1A3 and GAD1 on propofol susceptibility, and the molecular alterations for unconsciousness.

Owing to the significant individual differences of propofol, it was not easy for anesthesiologists to provide precise dose regimens for each patient. Hypotension is a common, sometimes even hazardous, adverse effect, particularly in patients with hypovolemia or requiring emergency procedures. We found that the $\mathrm{CC}$ genotype of GABRA2 rs11503014 decreased the risk of propofol-induced blood pressure reduction. GABRA2 rs11503014 had also been 
reported to be associated with the cardiovascular susceptibility of Chinese patients to propofol anesthesia. ${ }^{11}$ Since there was no direct evidence on the relationships between hypotension and GABA, further studies should pay more attention to the mechanism about the influences of polymorphisms in the GABAAR genes on blood pressure during propofol mediated anesthesia.

In addition to genetic factors, we found that gender might be an affecting factor of the LOC latency of patients anesthetized with propofol. Female required a shorter time to LOC than male, revealing that females were more sensitive to propofol than men. Steroids and sex hormones, such as progesterone, have sedative and anesthetic effects. $^{32,33}$ In the present study, anesthesia was administered for thyroid surgery and breast surgery; hence, a higher number of females than males were included. Multiple variable analysis was carried out to assess all the statistical significance, and gender was not consist in the final linear model. Besides, each genotype cohort was well balanced with respect to gender in our gene analysis, which avoided the influence of confounding factors.

To sum up, the results from this study indicated that genetic factors significantly influenced the patients' responses of propofol. However, a limited number of genes were included in the observation and analysis, therefore, subsequent studies should explore more gene polymorphisms and clinical indicators among a wider and diverse population.

\section{Conclusion}

In conclusion, this study showed that genetic polymorphisms of GABRA2, GABRB1, GABRG2, GAD1 and SLC1A3 had influence on LOC latency during propofol mediated total intravenous anesthesia in Chinese patients. GABRA2 rs11503014 was significantly associated with blood pressure reduction during anesthesia induction. Our findings suggested that in formulating individualized medication regimens with propofol, more attention should be paid to the effects of gene polymorphisms.

\section{Abbreviations}

TCI, Target-controlled infusion; LOC, Loss of consciousness; $\mathrm{Cp}$, target plasma concentration; GABAAR, Gamma-Aminobutyric Acid Type A Receptor; SLC1A3, Solute Carrier Family 1 Member 3; GAD, Glutamate Decarboxylase; SCN9A, Sodium Voltage-Gated Channel Alpha Subunit 9; DRD2, Dopamine Receptor D2; SNP, single nucleotide polymorphism; ASA, American Society of Anesthesiologists; SP, Systolic blood pressure; DP,
Diastolic blood pressure; MAP, Mean arterial pressure; GABRA2, Gamma-Aminobutyric Acid Type A Receptor Subunit Alpha 2; GABRB1, Gamma-Aminobutyric Acid Type A Receptor Subunit Beta 1; GABRG2, GammaAminobutyric Acid Type A Receptor Subunit Gamma 2.

\section{Data Sharing Statement}

The de-identified data of the current study are available from the corresponding authors (Zhongxing Wang, wzhxing@mail.sysu.edu.cn, or Jiali Li, lijiali5@mail. sysu.edu.cn) upon reasonable request. Data obtained in the results section can be shared, including correlation analysis between SNPs and propofol susceptibility, and multivariate linear analysis. No other additional unpublished documents are available apart from these data. We may balance the potential benefits and risks for each request and then provide the data that could be shared. Data will be available for 1 year after publication.

\section{Ethics Approval and Informed Consent}

The study complied with the Declaration of Helsinki protocol, was approved by the Ethics Committee of the First Affiliated Hospital of Sun Yat-sen University, Guangzhou, China, and was registered at the Chinese Clinical Trial Registry (ChiCTR1900022251). Written informed consent was obtained from all participants.

\section{Author Contributions}

All authors contributed to the conception, study design, execution, acquisition of data, analysis, and interpretation, or in all these areas; took part in drafting, revising or critically reviewing the article; have agreed on the journal to which the article will be submitted; gave final approval of the version to be published, and agree to be accountable for all aspects of the work.

\section{Funding}

This work was supported by the National Natural Science Foundation of China [Grants 81601711, 81971877], Key training programs for young teachers of Sun Yat-sen University [Grant 19ykzd12] and the Guangdong Provincial Key Laboratory of Construction Foundation [Grant 2017B030314030, 2020B1212060034].

\section{Disclosure}

The authors declare no conflicts of interest in this work. 


\section{References}

1. Sahinovic MM, Struys M, Absalom AR. Clinical pharmacokinetics and pharmacodynamics of propofol. Clin Pharmacokinet. 2018;57 (12):1539-1558. doi:10.1007/s40262-018-0672-3

2. Darracq M, Sleigh J, Banks MI, Sanders RD. Characterising the effects of propofol on complexity and stability in the EEG power spectrum. Brit J Anaesth. 2018;121(6):1368-1369. doi:10.1016/j. bja.2018.09.006

3. Glen JB. The development of 'Diprifusor': a TCI system for propofol. Anaesthesia. 1998;53(Suppl):13-21. doi:10.1111/j.13652044.1998.53s $115 . \mathrm{x}$

4. Hueppe T, Maurer F, Sessler DI, Volk T, Kreuer S. Retrospective comparison of Eleveld, Marsh, and Schnider propofol pharmacokinetic models in 50 patients. Brit $J$ Anaesth. 2020;124(2):E22-E24. doi:10.1016/j.bja.2019.10.019

5. Iohom G, Ni CM, O’brien JK, Cunningham AJ, Fitzgerald DF. An investigation of potential genetic determinants of propofol requirements and recovery from anaesthesia. Eur J Anaesthesiol. 2007;24 (11):912-919. doi:10.1017/S0265021507000476

6. Yang LQ, Li JJ, Chen SQ, Wang YW. Effect of different depths of anesthesia on perioperative stress response in children undergoing adenoidectomy and tonsillectomy. CNS Neurosci Ther. 2013;19 (2):134-135. doi:10.1111/cns.12038

7. Bowles TM, Freshwater-Turner DA, Janssen DJ, Peden CJ. Out-oftheatre tracheal intubation: prospective multicentre study of clinical practice and adverse events. Brit J Anaesth. 2011;107(5):687-692. doi:10.1093/bja/aer251

8. Concas A, Santoro G, Mascia MP, Serra M, Sanna E, Biggio G. The action of the general anesthetic propofol on GABAA receptors. $A d v$ Biochem Psychopharmacol. 1992;47:349-363.

9. Kotani Y, Shimazawa M, Yoshimura S, Iwama T, Hara H. The experimental and clinical pharmacology of propofol, an anesthetic agent with neuroprotective properties. CNS Neurosci Ther. 2008;14 (2):95-106. doi:10.1111/j.1527-3458.2008.00043.x

10. Li KY, Guan Y, Krnjevic K, Ye JH. Propofol facilitates glutamatergic transmission to neurons of the ventrolateral preoptic nucleus Anesthesiology. 2009;111(6):1271-1278. doi:10.1097/ALN.0b01 3e3181bf1d79

11. Zhong Q, Chen X, Zhao Y, Liu R, Yao S. Association of polymorphisms in pharmacogenetic candidate genes with propofol susceptibility. Sci Rep. 2017;7(1):3343. doi:10.1038/s41598-017-03229-3

12. Apfelbaum JL, Connis RT, Nickinovich DG, et al. Practice advisory for preanesthesia evaluation: an updated report by the American Society of Anesthesiologists Task Force on Preanesthesia Evaluation. Anesthesiology. 2012;116(3):522-538. doi:10.1097/ ALN.0b013e31823c1067

13. Marsh B, White M, Morton N, Kenny G. Pharmacokinetic model driven infusion of propofol in children. Br J Anaesth. 1991;67 (1):41-48. doi:10.1093/bja/67.1.41

14. Loparev VN, Cartas MA, Monken CE, Velpandi A, Srinivasan A. An efficient and simple method of DNA extraction from whole blood and cell lines to identify infectious agents. J Virol Methods. 1991;34 (1):105-112. doi:10.1016/0166-0934(91)90126-K

15. Enoch MA, Hodgkinson CA, Yuan Q, Shen PH, Goldman D, Roy A. The influence of GABRA2, childhood trauma, and their interaction on alcohol, heroin, and cocaine dependence. Biol Psychiatry. 2010;67 (1):20-27. doi:10.1016/j.biopsych.2009.08.019

16. Trucco EM, Villafuerte S, Heitzeg MM, Burmeister M, Zucker RA. Susceptibility effects of GABA receptor subunit alpha-2 (GABRA2) variants and parental monitoring on externalizing behavior trajectories: risk and protection conveyed by the minor allele. Dev Psychopathol. 2016;28(1):15-26. doi:10.1017/S0954579415000255
17. Sun Y, Zhang Y, Zhang D, et al. GABRA2 rs279858-linked variants are associated with disrupted structural connectome of reward circuits in heroin abusers. Transl Psychiatry. 2018;8(1):138. doi:10.1038/s41398018-0180-0

18. Haerian BS, Baum L, Kwan P, et al. Contribution of GABRG2 polymorphisms to risk of epilepsy and febrile seizure: a multicenter cohort study and meta-analysis. Mol Neurobiol. 2016;53 (8):5457-5467. doi:10.1007/s12035-015-9457-y

19. Kirenskaya AV, Storozheva ZI, Gruden MA, Sewell R. COMT and GAD1 gene polymorphisms are associated with impaired antisaccade task performance in schizophrenic patients. Eur Arch Psychiatry Clin Neurosci. 2018;268(6):571-584. doi:10.1007/s00406-018-0881-7

20. Shi Y, Li Y, Zhang J, Xiao Y, Yan P, Zhu Y. GAD1 but not GAD2 polymorphisms are associated with heroin addiction phenotypes. Neurosci Lett. 2020;717:134704. doi:10.1016/j.neulet.2019.134704

21. Huang X, Zhang Q, Chen X, Gu X, Wang M, Wu J. A functional variant in SLC1A3 influences ADHD risk by disrupting a hsa-miR3171 binding site: a two-stage association study. Genes Brain Behav. 2019;18(5):e12574. doi:10.1111/gbb.12574

22. Ying SW, Goldstein PA. Propofol suppresses synaptic responsiveness of somatosensory relay neurons to excitatory input by potentiating GABA(A) receptor chloride channels. Mol Pain. 2005;1:2. doi:10.1186/1744-8069-1-2

23. Murugaiah KD, Hemmings HJ. Effects of intravenous general anesthetics on $[3 \mathrm{H}] \mathrm{GABA}$ release from rat cortical synaptosomes. Anesthesiology. 1998;89(4):919-928. doi:10.1097/00000542-199810000-00017

24. Kasaragod VB, Schindelin H. Structure of heteropentameric GABA(A) receptors and receptor-anchoring properties of gephyrin. Front Mol Neurosci. 2019;12:191. doi:10.3389/fnmol.2019.00191

25. Franks NP. General anaesthesia: from molecular targets to neuronal pathways of sleep and arousal. Nat Rev Neurosci. 2008;9(5):370-386. doi:10.1038/nrn2372

26. Moyer RA, Wang D, Papp AC, et al. Intronic polymorphisms affecting alternative splicing of human dopamine D2 receptor are associated with cocaine abuse. Neuropsychopharmacol. 2011;36 (4):753-762. doi:10.1038/npp.2010.208

27. Crist RC, Clarke TK, Ang A, et al. An intronic variant in OPRD1 predicts treatment outcome for opioid dependence in African-Americans. Neuropsychopharmacol. 2013;38(10):2003-2010. doi:10.1038/ npp.2013.99

28. van Kuilenburg AB, Meijer J, Mul AN, et al. Intragenic deletions and a deep intronic mutation affecting pre-mRNA splicing in the dihydropyrimidine dehydrogenase gene as novel mechanisms causing 5-fluorouracil toxicity. Hum Genet. 2010;128(5):529-538. doi:10.1007/s00439-010-0879-3

29. Zhou Y, Danbolt NC. Glutamate as a neurotransmitter in the healthy brain. J Neural Transm. 2014;121(8):799-817. doi:10.1007/s00702014-1180-8.

30. Lee S, Lee Y, Lee GH. The regulation of glutamic acid decarboxylases in GABA neurotransmission in the brain. Arch Pharm Res. 2019;42(12):1031-1039. doi:10.1007/s12272-019-01196-z

31. Du J, Duan S, Wang H, et al. Comprehensive analysis of polymorphisms throughout GAD1 gene: a family-based association study in schizophrenia. J Neural Transm. 2008;115(3):513-519. doi:10.1007/ s00702-007-0844-z

32. Carl P, Hogskilde S, Nielsen JW, et al. Pregnanolone emulsion. A preliminary pharmacokinetic and pharmacodynamic study of a new intravenous anaesthetic agent. Anaesthesia. 1990;45 (3):189-197. doi:10.1111/j.1365-2044.1990.tb14683.x

33. Bitran D, Purdy RH, Kellogg CK. Anxiolytic effect of progesterone is associated with increases in cortical allopregnanolone and GABAA receptor function. Pharmacol Biochem Behav. 1993;45(2):423-428. doi:10.1016/0091-3057(93)90260-Z 


\section{Publish your work in this journal}

Pharmacogenomics and Personalized Medicine is an international, peer-reviewed, open access journal characterizing the influence of genotype on pharmacology leading to the development of personalized treatment programs and individualized drug selection for improved safety, efficacy and sustainability. This journal is indexed on the American Chemical Society's Chemical Abstracts Service (CAS). The manuscript management system is completely online and includes a very quick and fair peer-review system, which is all easy to use. Visit http://www.dovepress.com/testimonials.php to read real quotes from published authors. 